\title{
Germinação de sementes de Maclura tinctoria (Moraceae) sob diferentes regimes térmicos influenciados pela luz
}

\author{
Marina Crestana Guardia ${ }^{1,3}$, Edmir Vicente Lamarca $^{2}$
}

Recebido: 19.07.2012; aceito: 30.04 .2013

\begin{abstract}
Seeds germination of Maclura tinctoria (Moraceae) under different thermal regimes influenced by light). This study aimed to characterize germination of Maclura tinctoria seeds under different thermal regimes and alterations due presence or absence of light. Seeds were collected in Rio Claro, Ajapi, Itirapina, and Serra Negra, São Paulo State, Brazil. The results demonstrated that, in presence of light, seeds germinate in a wide temperature range $\left(15\right.$ to $\left.35^{\circ} \mathrm{C}\right)$ and better perform between 20 and $30^{\circ} \mathrm{C}$. The ideal temperature for seed germination was $30^{\circ} \mathrm{C}$. In the absence of light, germination percentage decreased as the temperature diminished and germination temperature range was narrowed, changing the ideal temperature for germination, showing that the seeds of Maclura tinctoria require presence of light to express their maximum germination rate. Key words: forest seeds, storage, taiúva
\end{abstract}

RESUMO - (Germinação de sementes de Maclura tinctoria (Moraceae) sob diferentes regimes térmicos influenciados pela luz). Este estudo teve como objetivo caracterizar a germinação de sementes de Maclura tinctoria sob diferentes regimes térmicos e alterações decorrentes da presença e ausência de luz. As sementes foram obtidas em Rio Claro, Ajapi, Itirapina e Serra Negra, SP. Os resultados demonstraram que na presença de luz as sementes germinam em ampla faixa térmica (15 a $35^{\circ} \mathrm{C}$ ) e apresentam melhor desempenho entre 20 e $30^{\circ} \mathrm{C}$, sendo $30^{\circ} \mathrm{C}$ a temperatura ideal para a germinação. Na ausência de luz a germinação decresceu à medida que a temperatura diminuiu e a faixa térmica germinativa se estreitou, alterando também a temperatura ideal para a germinação, mostrando que as sementes de Maclura tinctoria necessitam de luz para expressar a taxa máxima de germinação.

Palavras-chave: armazenamento, sementes florestais, taiúva

\section{Introdução}

Diversas sementes de espécies florestais do Brasil germinam em ampla faixa térmica e parecem apresentar relação positiva entre a temperatura ótima e o regime térmico da região de sua ocorrência natural (Borghetti 2005). Geralmente a faixa térmica adequada para a germinação de sementes de espécies florestais está entre 20 e $30{ }^{\circ} \mathrm{C}$ (Mello \& Barbedo 2007, Stockman et al. 2007, Coelho et al. 2008, Cardoso \& Pereira 2009, Pires et al. 2009, Pimenta et al. 2010, Lamarca et al. 2011). Contudo, podem ocorrer variações dentro da mesma espécie, em função das condições do ambiente de formação das sementes (Lamarca et al. 2011).

A temperatura é um fator de grande importância na germinação das sementes, podendo ser expressa em termos de temperaturas ótimas ou extremas e atua no percentual de germinação e desenvolvimento da espécie (Labouriau 1983, Malavasi 1988). Pode também estar associada às características adaptativas e ecológicas da espécie (Daws et al. 2004, Mattana et al. 2012).

Além da temperatura, a germinação das sementes pode depender de outros fatores ambientais, tais como a luz, a disponibilidade hídrica e de oxigênio (Mayer \& Poljakoff-Mayber 1989). A luz é um fator de extrema importância na germinação e no estabelecimento de plântulas no seu habitat natural (Yu et al. 2008). Sob o ponto de vista adaptativo e ecológico parece haver uma relação entre a exigência de luz e o tamanho da semente, em resposta da espécie ao habitat (Hewitt 1998).

1. Instituto de Botânica, Núcleo de Pesquisa em Sementes, Av. Miguel Stéfano, 3687, 04301-902 São Paulo, SP, Brasil

2. Programa de Pós-Graduação em Biodiversidade Vegetal e Meio Ambiente, Instituto de Botânica, Av. Miguel Stéfano, 3687, 04301-902 São Paulo, SP, Brasil

3. Autor para correspondência:m.c.guardia@gmail.com 
A percepção da luz pelas plantas ocorre através do fitocromo que corresponde à classe de pigmentos constituídos de cinco formas, sendo o fitocromo B o responsável pela indução da germinação em sementes (Takaki 2001).

Maclura tinctoria (L.) D.Don. ex Steud., conhecida popularmente como taiúva ou moreira, ocorre desde a região amazônica até o sul do país, porém é encontrada mais frequentemente em formações secundárias e matas abertas (Carvalho 2003). Pertence à família Moraceae, predominantemente tropical, e representada aproximadamente, por 1.500 espécies (Barroso et al. 2002). A espécie apresenta propriedade medicinal e os extratos elaborados a base de sua madeira e casca possuem atividades antibacteriana e antioxidante (Lamounier et al. 2012). Seus frutos têm forma oblonga, formados de núculas comprimidas, pericarpos carnosos e doces, indeiscentes, e de coloração amarelo-esverdeada quando maduros. A semente madura é achatada lateralmente, ligeiramente ovalada, com coloração creme e superfície lisa, formando plântulas classificadas como epígeafoliácea, apresentando elevada taxa de germinação (Battilani et al. 2006). Além disso, a espécie se desenvolve adequadamente na condição de luz ou sombreamento, na fase inicial do crescimento (Almeida et al. 2005).

Embora a espécie apresente ampla distribuição no Brasil e pertença a uma família de grande representatividade e importância ecológica, existem poucas informações na literatura científica sobre a germinação de suas sementes. Sendo assim, o presente estudo teve como objetivo caracterizar a germinação de sementes de $M$. tinctoria sob diferentes regimes térmicos e as alterações decorrentes da presença ou ausência de luz.

\section{Material e métodos}

As sementes de Maclura tinctoria foram obtidas de frutos maduros, recém dispersos (coletados logo após a queda sobre lona plástica) de cerca de 10 matrizes localizadas nas regiões de Rio Claro $\left(22^{\circ} 17^{\prime} \mathrm{S}\right.$, $\left.47^{\circ} 30^{\prime} \mathrm{O}\right)$, Ajapi $\left(22^{\circ} 17^{\prime} \mathrm{S}, 47^{\circ} 32^{\prime} \mathrm{O}\right)$, Itirapina $\left(22^{\circ} 25^{\prime} \mathrm{S}\right.$, $\left.47^{\circ} 34^{\prime} \mathrm{O}\right)$ e Serra Negra $\left(22^{\circ} 36^{\prime} \mathrm{S}, 46^{\circ} 36^{\prime} \mathrm{O}\right)$, no Estado de São Paulo, nos anos de 2004 e 2007 (tabela 1). As sementes foram extraídas manualmente dos frutos, macerados em peneira sob água corrente. A mistura foi separada por decantação, sendo as sementes mais densas que os tecidos dos frutos. Em seguida as sementes foram armazenadas em câmara fria a $7^{\circ} \mathrm{C}$, até o início dos experimentos, não excedendo 30 dias após a coleta. As sementes foram caracterizadas quanto ao teor de água e conteúdo de massa de matéria seca, determinados gravimetricamente pelo método de estufa a $105{ }^{\circ} \mathrm{C}$ por 24 horas (Brasil 2009), utilizando-se 4 repetições de 100 sementes. Os resultados foram apresentados em porcentagem, em base úmida, para o teor de água e em mg semente ${ }^{-1}$, para a massa de matéria seca.

Para o estudo da influência da temperatura e da luz, os testes de germinação foram conduzidos em câmaras do tipo B.O.D. As sementes foram dispostas em placas de Petri com papel filtro umedecido com água destilada (2,5 vezes o peso seco do papel), com duas folhas para a base e uma para a cobertura (Brasil 2009).

Inicialmente, sementes recém dispersas, oriundas de Rio Claro, Ajapi, Itirapina e Serra Negra (tabela 1) foram colocadas para germinar sob diferentes temperaturas $\left(15,20,25,30\right.$ e $\left.35^{\circ} \mathrm{C}\right)$, na presença e ausência de luz, em esquema fatorial. Para a condição de ausência de luz, as placas de Petri foram colocadas em caixas pretas do tipo gerbox (Araújo Neto et al. 2002).

Tabela 1. Procedência, época de coleta, classificação climática de Köppen, teor de água e massa de matéria seca das sementes de Maclura tinctoria recém dispersas, de diferentes localidades do Estado de São Paulo.

Table 1. Origin, harvest time, Köppen climate classification, water content, and seed dry mass of Maclura tinctoria recently dispersed at different locations in the State of São Paulo, Brazil.

\begin{tabular}{lccc}
\hline $\begin{array}{l}\text { Procedência e época de coleta } \\
\text { (latitude, longitude, altitude) }\end{array}$ & Classificação de Köppen & $\begin{array}{c}\text { Teor de água } \\
(\%, \text { base úmida })\end{array}$ & $\begin{array}{c}\text { Massa seca } \\
\left(\mathrm{mg} \mathrm{semente}^{-1}\right)\end{array}$ \\
\hline $\begin{array}{l}\text { Rio Claro, SP - Nov/2007 } \\
\left(22^{\circ} 17^{\prime} \mathrm{S}, 47^{\circ} 30^{\prime} \mathrm{O}, 620 \mathrm{~m}\right)\end{array}$ & Cwa & 7,97 & 2,74 \\
$\begin{array}{l}\text { Ajapi, SP - Nov/2007 } \\
\left(22^{\circ} 17^{\prime} \mathrm{S}, 47^{\circ} 32^{\prime} \mathrm{O}, 675 \mathrm{~m}\right)\end{array}$ & Cwa & 7,53 & 2,80 \\
$\begin{array}{l}\text { Itirapina, SP - Nov/2004 } \\
\left(22^{\circ} 25^{\prime} \mathrm{S}, 47^{\circ} 34^{\prime} \mathrm{O}, 772 \mathrm{~m}\right)\end{array}$ & Cwa & 8,18 & 2,69 \\
$\begin{array}{l}\text { Serra Negra, SP - Nov/2007 } \\
\left(22^{\circ} 36^{\prime} \mathrm{S}, 46^{\circ} 36^{\prime} \mathrm{O}, 1014 \mathrm{~m}\right)\end{array}$ & Cwa & 8,49 & 2,77 \\
\hline
\end{tabular}


Posteriormente, trabalhou-se apenas com sementes de $M$. tinctoria coletadas em Itirapina no ano de 2004, depois de permanecerem armazenadas por 9 e 28 meses em câmara fria a $7{ }^{\circ} \mathrm{C}$, em frascos herméticos e na ausência de luz. Após cada período de armazenamento, as sementes foram colocadas para germinar sob 25 e $30{ }^{\circ} \mathrm{C}$, na presença ou ausência de luz, conforme descrito anteriormente. Comparou-se ainda a germinação das sementes recém dispersas com as armazenadas, nas mesmas condições. Foi analisada também, para as sementes recém dispersas, a influência da quantidade de luz na germinação. Para tanto, as sementes foram colocadas para germinar sob fotoperíodo de 12 horas na presença ou ausência de luz, nas temperaturas de 25 ou $30^{\circ} \mathrm{C}$.

As avaliações da germinação foram realizadas diariamente, durante 30 dias, sendo registradas as sementes que emitiram raiz primária. Foram calculados a porcentagem de germinação, o tempo médio de germinação e a variância do tempo médio de germinação (Borghetti \& Ferreira 2004). Na ausência de luz, as avaliações foram realizadas sob luz verde de segurança (Amaral-Baroli \& Takaki 2001).

O delineamento experimental e o procedimento estatístico foi inteiramente casualizado, em esquema fatorial associando-se temperatura e luz, $5 \times 2$, separadamente para sementes coletadas nas regiões de Rio Claro, Ajapi, Itirapina, e Serra Negra; temperatura e luz, $2 \times 2$, separadamente para sementes recém dispersas e armazenadas por 9 e 28 meses; e temperatura e luz, $2 \times 3$, apenas para sementes recém dispersas. Para todas as análises foram utilizadas 4 repetições de 25 sementes. Os dados obtidos foram submetidos à análise de variância (teste F), ao nível de 5\% de probabilidade. Quando pertinente, as médias foram comparadas entre si pelo teste de Tukey ao nível de 5\% (Santana \& Ranal 2004).

\section{Resultados e discussão}

As análises de variância dos dados de porcentagem de germinação, tempo médio e variância do tempo médio de germinação apresentaram interação significativa entre os fatores temperatura e luz para as sementes de Maclura tinctoria coletadas em Rio Claro, Ajapi, Itirapina e Serra Negra (tabela 2), apenas não houve interação para as variáveis de variância do tempo médio e de tempo médio de germinação, respectivamente, quando as sementes foram coletadas em Rio Claro e Serra Negra (tabela 2).
Os resultados de germinação demonstraram que na presença de luz as sementes de $M$. tinctoria, procedentes de Rio Claro, Ajapi, Itirapina e Serra Negra, germinam na faixa térmica de 15 a $35{ }^{\circ} \mathrm{C}$ (tabela 2). No entanto, quando analisada a variável de germinação em conjunto com o tempo médio e com a variância do tempo médio, nota-se que a faixa térmica se estreita para 20 a $30{ }^{\circ} \mathrm{C}$. Verifica-se ainda que $30{ }^{\circ} \mathrm{C}$ foi a temperatura ideal e comum para todas as procedências e variáveis, ou seja, condição na qual as sementes apresentaram os maiores valores de germinação e o melhor desempenho em tempo, germinando de forma mais rápida e homogênea (tabela 2).

A faixa térmica germinativa de 20 a $30{ }^{\circ} \mathrm{C}$ pode ser considerada adequada para as sementes de $M$. tinctoria, algo que parece se manter independente da origem do material, corroborando as faixas térmicas encontradas para diversas espécies florestais (Miranda \& Ferraz 1999, Santos et al. 2004, Mello \& Barbedo 2007, Stockman et al. 2007, Coelho et al. 2008, Cardoso \& Pereira 2009, Pires et al. 2009, Pimenta et al. 2010, Lamarca et al. 2011).

$\mathrm{Na}$ ausência de luz, a faixa térmica germinativa para as sementes de $M$. tinctoria foi mais estreita que na presença de luz (tabela 2). Para sementes procedentes de Itirapina, por exemplo, não ocorreu germinação a $15{ }^{\circ} \mathrm{C}$. Em geral, as porcentagens de germinação foram mais baixas e o tempo médio de germinação, bem como a variância do tempo médio, aumentaram à medida que a temperatura diminuiu. Por outro lado, na temperatura mais elevada $\left(35^{\circ} \mathrm{C}\right)$ a germinação foi igual tanto na presença quanto na ausência de luz, exceto para as sementes procedentes de Ajapi (tabela 2). As sementes de Serra Negra apresentaram, aproximadamente, $30 \%$ de germinação sob $15{ }^{\circ} \mathrm{C}$, valor alcançado para as sementes de Ajapi e Itirapina, respectivamente, apenas sob 20 e $30{ }^{\circ} \mathrm{C}$, sendo que a germinação foi praticamente nula na ausência de luz sob $15{ }^{\circ} \mathrm{C}$ para sementes dessas procedências. Ainda quanto às procedências de Ajapi e Itirapina, observa-se que na temperatura de $30{ }^{\circ} \mathrm{C}$, anteriormente identificada como ideal para a germinação de $M$. tinctoria das quatro procedências, não foi suficiente para que as sementes de Ajapi e Itirapina apresentassem bom desempenho germinativo na ausência de luz (tabela 2). Sendo assim, nossos resultados sugerem que as sementes de $M$. tinctoria necessitam de luz para expressar seu máximo de germinação.

Os resultados de germinação, no gradiente térmico, permitiram identificar distinções quanto 
Tabela 2. Germinação, tempo médio de germinação e variância do tempo médio de germinação de sementes de Maclura tinctoria em função da temperatura e da luz. Sementes procedentes de Rio Claro, Ajapi, Itirapina e Serra Negra, SP. Médias seguidas pela mesma letra (minúsculas nas colunas, maiúsculas nas linhas) não diferem entre si pelo teste de Tukey a $5 \%$.

Table 2. Germination, germination time and, variance of the average germination time of Maclura tinctoria seed related to temperature and light. Seeds collected in Rio Claro, Ajapi, Itirapina and Serra Negra, State of São Paulo, Brazil. Means followed by the same letter (lowercase in columns, capital letters in lines) do not differ by Tukey test at 5\%.

\begin{tabular}{|c|c|c|c|c|c|c|c|c|}
\hline \multirow{2}{*}{$\begin{array}{l}\text { Temperatura } \\
\left({ }^{\circ} \mathrm{C}\right)\end{array}$} & \multicolumn{2}{|c|}{ Germinação (\%) } & \multicolumn{3}{|c|}{ Tempo médio de germinação } & \multicolumn{3}{|c|}{ Variância do tempo médio } \\
\hline & Luz & \multirow[t]{2}{*}{ Escuro } & \multirow[t]{2}{*}{ Luz } & \multicolumn{2}{|l|}{ Escuro } & \multirow[t]{2}{*}{ Luz } & \multicolumn{2}{|l|}{ Escuro } \\
\hline Rio Claro & & & & & Médias & & & Médias \\
\hline 15 & $65 \mathrm{aA}$ & $18 \mathrm{cdB}$ & $11,8 \mathrm{aB}$ & $16,8 \mathrm{aA}$ & & 4,5 & 13,3 & $8,9 \mathrm{a}$ \\
\hline 20 & $68 \mathrm{aA}$ & $11 \mathrm{~dB}$ & $10,4 \mathrm{abA}$ & $10,5 \mathrm{bA}$ & & 7,6 & 1,4 & $4,5 \mathrm{a}$ \\
\hline 25 & $65 \mathrm{aA}$ & $46 \mathrm{bcA}$ & 8,3 bA & $9,4 \mathrm{bA}$ & & 0,7 & 4,9 & $2,8 \mathrm{a}$ \\
\hline 30 & $75 \mathrm{aA}$ & $80 \mathrm{aA}$ & $4,1 \mathrm{cB}$ & $8,8 \mathrm{bA}$ & & 5,5 & 6,9 & $6,2 \mathrm{a}$ \\
\hline 35 & $46 \mathrm{aA}$ & $55 \mathrm{abA}$ & $8,0 \mathrm{bA}$ & $9,1 \mathrm{bA}$ & & 8,9 & 12,0 & $10,5 \mathrm{a}$ \\
\hline \multicolumn{3}{|l|}{ Médias } & & & & $5,4 \mathrm{~A}$ & 7,7 A & \\
\hline \multicolumn{3}{|l|}{ Coeficiente de Variação } & \multicolumn{2}{|c|}{$14,39 \%$} & & & \\
\hline \multicolumn{3}{|l|}{ Ajapi } & & & Médias & & & Médias \\
\hline 15 & $53 \mathrm{bA}$ & $1 \mathrm{cB}$ & 15,9 aA & $4,0 \mathrm{~dB}$ & & 5,9 aA & $0,0 \mathrm{bB}$ & \\
\hline 20 & $97 \mathrm{aA}$ & $36 \mathrm{abB}$ & $11,7 \mathrm{abB}$ & 17,9 aA & & $2,2 \mathrm{aB}$ & $8,9 \mathrm{aA}$ & \\
\hline 25 & $98 \mathrm{aA}$ & $48 \mathrm{aB}$ & $8,6 \mathrm{bcB}$ & $14,0 \mathrm{abA}$ & & $1,3 \mathrm{aA}$ & $4,0 \mathrm{abA}$ & \\
\hline 30 & $97 \mathrm{aA}$ & $44 \mathrm{aB}$ & $4,6 \mathrm{cA}$ & $5,6 \mathrm{cdA}$ & & $1,8 \mathrm{aA}$ & $6,6 \mathrm{abA}$ & \\
\hline 35 & $44 \mathrm{bA}$ & $20 \mathrm{bcB}$ & $12,4 \mathrm{abA}$ & $10,8 \mathrm{bcA}$ & & $6,4 \mathrm{aA}$ & 7,7 aA & \\
\hline \multicolumn{9}{|l|}{ Médias } \\
\hline \multicolumn{2}{|l|}{ Coeficiente de Variação } & & 28,6 & & & 78,2 & $0 \%$ & \\
\hline Itirapina & & & & & Médias & & & Médias \\
\hline 15 & $84 \mathrm{bA}$ & $0 \mathrm{cB}$ & $19,4 \mathrm{aA}$ & $0,0 \mathrm{bB}$ & & $9,4 \mathrm{aA}$ & $0,0 \mathrm{bA}$ & \\
\hline 20 & $98 \mathrm{abA}$ & $5 \mathrm{cB}$ & $11,2 \mathrm{bA}$ & $11,0 \mathrm{aA}$ & & $2,6 \mathrm{aA}$ & $14,5 \mathrm{abA}$ & \\
\hline 25 & $90 \mathrm{abA}$ & $10 \mathrm{cB}$ & $8,4 \mathrm{bA}$ & $10,1 \mathrm{aA}$ & & $5,4 \mathrm{aB}$ & 37,7 aA & \\
\hline 30 & $100 \mathrm{aA}$ & $49 \mathrm{bB}$ & $6,4 \mathrm{bA}$ & $6,5 \mathrm{aA}$ & & $1,9 \mathrm{aA}$ & $15,2 \mathrm{abA}$ & \\
\hline 35 & $97 \mathrm{abA}$ & $86 \mathrm{aA}$ & $6,5 \mathrm{bA}$ & $7,1 \mathrm{aA}$ & & $2,7 \mathrm{aA}$ & $3,3 \mathrm{bA}$ & \\
\hline Médias & & & & & & & & \\
\hline Coeficiente de Variaçã & 12,3 & & 29,9 & & & 134, & $6 \%$ & \\
\hline Serra Negra & & & & & Médias & & & Médias \\
\hline 15 & $82 \mathrm{abA}$ & $32 \mathrm{bB}$ & 14,9 & 17,1 & $16,1 \mathrm{a}$ & $10,3 \mathrm{aA}$ & $7,2 \mathrm{bA}$ & \\
\hline 20 & $76 \mathrm{abA}$ & $93 \mathrm{aA}$ & 10,6 & 11,6 & $11,1 \mathrm{~b}$ & $2,4 \mathrm{bB}$ & $9,7 \mathrm{bA}$ & \\
\hline 25 & 96 aA & $95 \mathrm{aA}$ & 8,7 & 9,6 & $9,3 \mathrm{~b}$ & $3,0 \mathrm{bA}$ & $4,2 \mathrm{bA}$ & \\
\hline 30 & $95 \mathrm{aA}$ & $96 \mathrm{aA}$ & 4,5 & 4,8 & $4,7 \mathrm{c}$ & $3,0 \mathrm{bA}$ & $2,8 \mathrm{bA}$ & \\
\hline 35 & $60 \mathrm{bA}$ & $80 \mathrm{aA}$ & 9,8 & 8,7 & $9,2 \mathrm{~b}$ & $8,4 \mathrm{abB}$ & $17,2 \mathrm{aA}$ & \\
\hline Médias & & & $9,8 \mathrm{~A}$ & $10,4 \mathrm{~A}$ & & & & \\
\hline Coeficiente de Variaçâ & 20, & & 17,8 & & & 52,4 & $1 \%$ & \\
\hline
\end{tabular}


Tabela 3. Germinação, tempo médio de germinação e variância do tempo médio de germinação em função da temperatura e da luz de sementes recém dispersas de Maclura tinctoria procedentes de Itirapina, SP (coletadas em 2004). Fotoperíodo de 12 horas, FOT; luz constante, LUZ; ausência de luz, ESC. Médias seguidas pela mesma letra (minúsculas nas colunas, maiúsculas nas linhas) não diferem entre si pelo teste de Tukey a $5 \%$.

Table 3. Germination, average germination time, and variance of the average germination time of recently dispersed seeds of Maclura tinctoria in function of temperature and light. Seeds collected in Itirapina, State of São Paulo, Brazil (collected in 2004). Photoperiod of 12 hours, FOT; constant light, LUZ; absence of light, ESC. Means followed by the same letter (lowercase in columns, capital letters in lines) do not differ by Tukey test at $5 \%$.

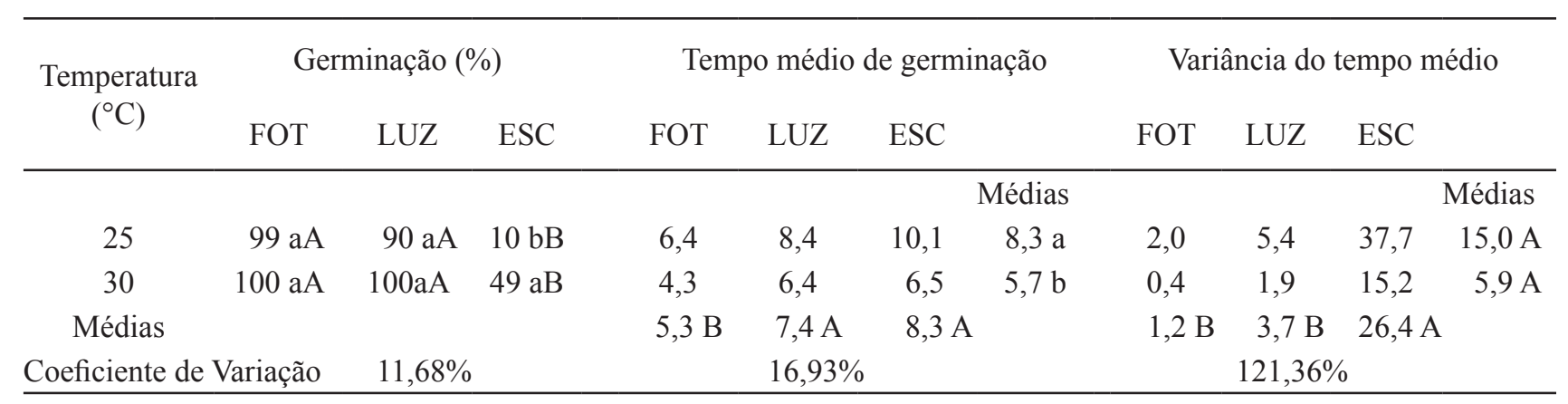

à exigência luminosa, entre as procedências. Tais distinções podem ser reflexos da variabilidade genética das sementes ou das condições do ambiente de formação, uma vez que diversas características físicas e fisiológicas das sementes, como o teor de água inicial, os níveis de dormência e as exigências térmicas e fotoblásticas, podem ser influenciadas por variáveis meteorológicas, tais como temperatura, precipitação pluvial e fotoperíodo durante a formação das sementes (Laboriau 1983, Cardoso et al. 1995, Daws et al. 2004, 2006, Martins et al. 2009, Lamarca et al. 2011). Por outro lado, diversas características fisiológicas, principalmente o fotoblastismo, podem ter origem na herdabilidade genética das sementes, ou seja, seriam pouco sujeitas às variações do ambiente durante o desenvolvimento (Cardoso et al. 1995).

A exigência de luz para a germinação, para o tempo médio de germinação e para a variância do tempo médio de germinação das sementes de M. tinctoria ocorreu independente da quantidade de luz fornecida (tabela 3 ). Ao comparar-se a germinação sob $25{ }^{\circ} \mathrm{C}$ e $30{ }^{\circ} \mathrm{C}$ na presença de luz constante e no fotoperíodo de 12 horas, verificou-se, para as sementes de Itirapina, que os valores foram em média de $95 \%$ para luz constante e de $100 \%$ para 12 horas de fotoperíodo, ou seja, não diferindo significativamente. Já na ausência de luz a germinação sob $25^{\circ} \mathrm{C}$ foi de $10 \%$ e sob $30{ }^{\circ} \mathrm{C}$ foi de $49 \%$, ambos diferindo das condições anteriores (tabela 3 ). Essa combinação de luz e temperatura e a influência na germinação foi observada também por Galindo et al. (2012), em sementes de Crataeva tapia L.
A relação luz e temperatura na germinação de sementes de $M$. tinctoria foi verificada para as sementes recém coletadas e armazenadas por 9 e 28 meses (tabela 4). A análise de variância apresentou interação significativa entre os fatores temperatura e luz para a variável de germinação, indiferente do tempo de armazenamento. Verificou-se que após 9 meses de armazenamento, sob $30^{\circ} \mathrm{C}$, não houve diferença significativa entre presença ou ausência de luz, algo que havia ocorrido para as sementes recém dispersas. Após 28 meses de armazenamento os valores de germinação sob $25{ }^{\circ} \mathrm{C}$ não diferiram significativamente entre as condições de luz, algo que ocorreu sob $30^{\circ} \mathrm{C}$ para as sementes de Maclura tinctoria (tabela 4).

A percepção da luz pelas plantas ocorre através do fitocromo, que corresponde a uma classe de pigmentos constituídos de cinco formas distintas. O fitocromo B é o responsável pela indução da germinação de sementes (Takaki 2001). Alguns fatores como a idade das sementes, o tempo de armazenamento, tratamentos para superar dormência, estresse hídrico, bem como a quantidade e qualidade de luz na germinação podem alterar a sensibilidade da semente ao regime luminoso, induzindo ou inibindo a germinação (Bewley \& Black 1994). Essas respostas foram observadas por diversos autores, tais como: Socolowski \& Takaki (2004) e Ferraz-Grande \& Takaki (2006), quando estudaram estresse hídrico e perda da sensibilidade à luz, respectivamente em sementes de Jacaranda mimosifolia D.Don e Caesalpinia pelthoforoides Benth.; Godoi \& Takaki (2005) e Godoi et al. (2009), quando estudaram a qualidade e quantidade 
Tabela 4. Germinação, tempo médio de germinação e variância do tempo médio de germinação em função da temperatura e da luz de sementes de Maclura tinctoria procedentes de Itirapina, SP. Sementes recém dispersas, armazenadas por 9 e 28 meses. Médias seguidas pela mesma letra (minúsculas nas colunas, maiúsculas nas linhas) não diferem entre si pelo teste de Tukey, a 5\%.

Table 4. Germination, average germination time, and variance of the average germination time as a function of temperature and light of Maclura tinctoria seeds collected in Itirapina, State of São Paulo, Brazil. Recently dispersed seeds, stored for 9 and 28 months. Means followed by the same letter (lowercase in columns, capital letters in lines) do not differ by Tukey test at $5 \%$.

\begin{tabular}{|c|c|c|c|c|c|c|c|c|}
\hline \multirow{2}{*}{ Temperatura $\left({ }^{\circ} \mathrm{C}\right)$} & \multicolumn{2}{|c|}{ Germinação (\%) } & \multicolumn{3}{|c|}{ Tempo médio de germinação } & \multicolumn{3}{|c|}{ Variância do tempo médio } \\
\hline & Luz & Escuro & Luz & Escuro & & Luz & Escuro & \\
\hline Recém dispersas & & & & & Médias & & & Médias \\
\hline 25 & $99 \mathrm{aA}$ & $10 \mathrm{bB}$ & 6,4 & 10,1 & $8,3 \mathrm{a}$ & 2,0 & 37,7 & $19,8 \mathrm{a}$ \\
\hline 30 & $100 \mathrm{aA}$ & $49 \mathrm{aB}$ & 4,3 & 6,5 & $5,4 \mathrm{~b}$ & 0,4 & 15,2 & $7,8 \mathrm{a}$ \\
\hline Médias & & & $5,3 \mathrm{~B}$ & $8,3 \mathrm{~A}$ & & $1,2 \mathrm{~B}$ & $26,5 \mathrm{~A}$ & \\
\hline Coeficiente de Variação & \multicolumn{2}{|c|}{$11,67 \%$} & \multicolumn{2}{|c|}{$16,58 \%$} & & \multicolumn{2}{|c|}{$110,21 \%$} & \\
\hline Armazenadas por & & & & & Médias & & & Médias \\
\hline 25 & $100 \mathrm{aA}$ & $21 \mathrm{bB}$ & $7,4 \mathrm{aB}$ & $10,9 \mathrm{aA}$ & & 1,1 & 13,9 & $7,5 \mathrm{a}$ \\
\hline 30 & $98 \mathrm{aA}$ & $94 \mathrm{aA}$ & $4,7 \mathrm{bB}$ & $6,2 \mathrm{bA}$ & & 0,7 & 3,1 & $1,9 \mathrm{a}$ \\
\hline Médias & & & & & & $0,9 \mathrm{~A}$ & $8,5 \mathrm{~A}$ & \\
\hline Coeficiente de Variação & \multicolumn{2}{|c|}{$5,67 \%$} & \multicolumn{2}{|c|}{$7,94 \%$} & & \multicolumn{2}{|c|}{$158,14 \%$} & \\
\hline $\begin{array}{c}\text { Armazenadas por } \\
28 \text { meses }\end{array}$ & & & & & Médias & & & Médias \\
\hline 25 & $12 \mathrm{bA}$ & $13 \mathrm{aA}$ & 16,4 & 16,0 & $16,2 \mathrm{a}$ & 14,9 & 9,3 & $12,1 \mathrm{~A}$ \\
\hline 30 & $43 \mathrm{aA}$ & $22 \mathrm{aB}$ & 9,2 & 10,6 & $9,9 \mathrm{~b}$ & 8,2 & 13,9 & $11,1 \mathrm{~A}$ \\
\hline Médias & & & $12,8 \mathrm{~A}$ & $13,3 \mathrm{~A}$ & & $11,6 \mathrm{~A}$ & $11,6 \mathrm{~A}$ & \\
\hline Coeficiente de Variação & \multicolumn{2}{|c|}{$34,99 \%$} & \multicolumn{2}{|c|}{$13,32 \%$} & & \multicolumn{2}{|c|}{$98,55 \%$} & \\
\hline
\end{tabular}

espectral da luz e a sensibilidade na germinação, respectivamente, em sementes de Cecropia glaziovi Snethl. e Miconia theaezans (Bonpl.) Cogniaux; Araújo Neto et al. (2002), quando estudaram o armazenamento e a sensibilidade à luz em sementes de Guazuma ulmifolia Lam.; e Bufalo et al. (2012), quando estudaram a estratificação e a sensibilidade à luz em sementes de Lactuca sativa L.

Neste estudo observou-se que o período de armazenamento e principalmente o regime térmico para a germinação foram os fatores que influenciaram a sensibilidade à luz na germinação de Maclura tinctoria, visto que para essas sementes atingirem valores máximos de germinação existe a necessidade da combinação entre temperatura e luz, como por exemplo, a germinação sob baixas temperaturas com a presença de luz.

Por fim, os resultados demonstraram que as sementes de Maclura tinctoria, na presença de luz, germinam em ampla faixa térmica $\left(15\right.$ a $\left.35^{\circ} \mathrm{C}\right)$ apresentando maior desempenho na faixa de 20 a $30{ }^{\circ} \mathrm{C}$, sendo $30^{\circ} \mathrm{C}$ a temperatura considerada ideal para a germinação. $\mathrm{Na}$ ausência de luz, os valores de germinação e de desempenho decrescem à medida que a temperatura diminui, e dependendo da procedência das sementes, a faixa térmica germinativa torna-se mais estreita $\left(20\right.$ a $\left.35^{\circ} \mathrm{C}\right)$, alterando também a temperatura ideal, mostrando que as sementes de M. tinctoria necessitam de luz para expressar a taxa máxima de germinação.

\section{Agradecimentos}

Os autores agradecem ao Dr. Massanori Takaki e ao Dr. Claudio José Barbedo, pelas sugestões; à CAPES pela bolsa de doutorado concedida ao E.V. Lamarca; e ao Sr. Maurílio Antonio Alves Filho, pela autorização de coleta de sementes.

\section{Literatura citada}

Almeida, S.M.Z., Soares, A.M., Castro, E.M., Vieira, C.V. \& Gajego, E.B. 2005. Alterações morfológicas e alocação de espécies florestais sob diferentes condições de sombreamento. Ciência Rural 35: 62-68. 
Amaral-Baroli, A. \& Takaki, M. 2001. Phytochrome controls achene germination in Bidens pilosa L. (Asteraceae) by very low fluence response. Brazilian Archives of Biology and Technology 44: 121-124.

Araújo Neto, J.C., Aguiar, I.B., Ferreira, V.M. \& Rodrigues, T.J.D. 2002. Temperaturas cardeais e efeito da luz na germinação de sementes de mutamba. Revista Brasileira de Engenharia Agrícola e Ambiental 6: 460-465.

Barroso, G.M., Peixoto, A.L., Ichaso, C.L.F., Guimarães, E.F. \& Costa, C.G. 2002. Sistemática de angiospermas do Brasil. v.1. 2 ed. Universidade Federal de Viçosa, Viçosa.

Battilani, J.L., Santiago, E.F. \& Souza, A.L.T. 2006. Morfologia de frutos, sementes e desenvolvimento de plântulas e plantas jovens de Maclura tinctoria (L.) D.Don. ex Steud. (Moraceae). Acta Botanica Brasilica 20: 581-589.

Bewley, J.D. \& Black, M. 1994. Seeds: physiology of development and germination. Plenum Press, New York.

Borghetti, F. 2005. Temperaturas extremas e a germinação das sementes. In: R.J.M.C. Nogueira, E.L. Araújo, L.G. Willadino \& U.M.T. Cavalcante (orgs.). Estresses ambientais: danos e benefícios em plantas. Universidade Federal Rural de Pernambuco, Imprensa Universitária, Recife.

Borghetti, F. \& Ferreira, A.G. 2004. Interpretação de resultados de germinação. In: A.G. Ferreira \& F. Borghetti (eds.). Germinação: do básico ao aplicado. Artmed, Porto Alegre. pp. 209-222.

Brasil. 2009. Regras para análise de sementes. Ministério da Agricultura, Pecuária e Abastecimento, Brasília.

Bufalo, J., Amaro, A.C.E., Araújo, H.S., Corsato, J.M., Ono, E.O., Ferreira, G. \& Rodrigues, J.D. 2012. Períodos de estratificação na germinação de sementes de alface (Lactuca sativa L.) sob diferentes condições de luz e temperatura. Semina 33: 931-940.

Cardoso, V.J.M. 1995. Germinação e fotoblastismo de sementes de Cucumis anguria: influência da qualidade da luz durante a maturação e secagem. Revista Brasileira de Fisiologia Vegetal 7: 75-80.

Cardoso, V.J.M. \& Pereira, F.J.M. 2009. Dependência térmica da germinação de sementes de Drymaria cordata (L.) Willd. ex Roem. \& Schult. (Cariophyllaceae). Acta Botanica Brasilica 23: 305-312.

Carvalho, P.E.R. 2003. Espécies Arbóreas Brasileiras. Embrapa Florestas, Curitiba.

Coelho, M.F.B., Sales, D.M., Dombroski, J.L.D., Azevedo, R.A.B. \& Albuquerque, M.C.F. 2008. Condições de luz e temperatura na germinação de sementes de algodão do campo [Cochlospermum regium (Schrank) Pilger - Bixaceae]. Revista de Biologia Neotropical 5: 23-31.
Daws, M.I., Cleland, H., Chmielarz, P., Gorin, F., Leprince, O., Matthews, S., Mullins, C.E., Thanos, C.A., Vandvik, V. \& Pritchard, H.W. 2006. Variable dessication tolerance in Acer pseudoplatanus seeds in relation to developmental conditions: a case of phenotypic recalcitrance? Functional Plant Biology 33: 59-66.

Daws, M.I., Lydall, E., Chmielarz, P., Leprince, O., Matthews, S., Thanos, C.A. \& Pritchard, H.W. 2004. Developmental heat sum influences recalcitrant seed traits in Aesculus hippocastanum across Europe. New Phytologist 162: 157-166.

Ferraz-Grande, F.G.A. \& Takaki, M. 2006. Efeito da luz, temperatura e estresse de água na germinação de sementes de Caesalpinia peltophoroides Benth. (Caealpinoideae). Bragantia 65: 37-42.

Galindo, E.A., Alves, E.U., Bernado Silva, K.B., Barrozo, L.M., Silva, S. \& Moura, S. 2012. Germinação e vigor de sementes de Crataeva tapia $\mathrm{L}$. em diferentes temperaturas e regimes de luz. Revista Ciência Agronômica 43: 138-145.

Godoi, S. \& Takaki, M. 2005. Efeito da temperatura e a participação do fitocromo no controle da germinação de sementes de embaúba. Revista Brasileira de Sementes 27: 87-90.

Godoi, S., Grandis, A. \& Takaki, M. 2009. A germinação de sementes de Miconia theaezans (Bonpl.) Cogniaux (Melastomataceae) é controlada pelo fitocromo. Naturalia 32: 13-22.

Hewitt, N. 1998. Seed size and shade-tolerance: a comparative analysis of North American temperate trees. Oecologia 114: 432-440.

Labouriau, L.G. 1983. A germinação das sementes. OEA, Washington.

Lamarca, E.V., Silva, C.V. \& Barbedo, C.J. 2011. Limites térmicos para a germinação em função da origem de sementes de espécies de Eugenia (Myrtaceae) nativas do Brasil. Acta Botanica Brasilica 25: 293-300.

Lamounier, K.C., Cunha, L.C.S., Morais, S.A.L., Aquino, F.J.T., Chang, R., Nascimento, E.A., Souza, M.G.M., Martins, C.H.G. \& Cunha, W.R. 2012. Chemical analysis and study of phenolics, antioxidant activity, and antibacterial effect of the wood and bark of Maclura tinctoria (L.) D.Don ex Steud. Evidence-Based Complementary and Alternative Medicine, Article ID 451039, 7p. doi:10.1155/2012/451039.

Malavasi, M.M. 1988. Germinação de sementes. In: F.C.M. Pinã-Rodrigues (coord.). Manual de análise de sementes florestais. Fundação Cargill, Campinas, pp. 25-40.

Martins, C.C., Bovi, M.L.A., Nakagawa, J. \& Machado, C.G. 2009. Secagem e armazenamento de sementes de juçara. Revista Árvore 33: 635-642. 
Mattana, E., Daws, M.I., Fenu, G. \& Bacchetta, G. 2012. Adaptation to habitat in Aquilegia species endemic to Sardinia (Italy): Seed dispersal, germination and persistence in the soil. Plant Biosystems 146: 374-383.

Mayer, A.M. \& Poljakoff -Mayber, A. 1989. The Germination of Seeds. Pergamon Press, Oxford.

Mello, J.I.O. \& Barbedo, C.J. 2007. Temperatura, luz e substrato para a germinação de sementes de pau-brasil Caesalpinia echinata Lam., LeguminosaeCaesalpiniodeae. Revista Árvore 31: 645-655.

Miranda, P.R.M. \& Ferraz, I.D.K. 1999. Efeito da temperatura na germinação de sementes e morfologia da plântula de Maquira sclerophylla (Ducke) C.C. Berg. Revista Brasileira de Botânica 22: 303-307.

Pimenta, R.S., Luz, P.B., Pivetta, K.F.L., Castro, A. \& Pizetta, P.U.C. 2010. Efeito da maturação e temperatura na germinação de sementes de Phoenix canariensis Hort. ex Chabaud - Arecaceae. Revista Árvore 34: 31-38.

Pires, L.A., Cardoso, V.J.M., Joly, C.A. \& Rodrigues, R.R. 2009. Germinação de Ternstroemia brasiliensis Cambess. (Pentaphylacaceae) de Floresta de Restinga. Acta Botanica Brasilica 23: 57-66.
Santana, D.G. \& Ranal, M.A. 2004. Análise da germinação: um enfoque estatístico. Universidade de Brasília, Brasília.

Santos, C.M.R., Ferreira, A.G. \& Áquila, M.E.A. 2004. Características de frutos e germinação de sementes de seis espécies de Myrtaceae nativas do Rio Grande do Sul. Revista Ciência Florestal 14: 13-20.

Socolowski, F. \& Takaki, M. 2004. Germination of Jacaranda mimosifolia (D. Don - Bignoniaceae) seeds: effects of ligth, temperature and water stress. Brazilian Archives of Biology and Techonology 47: 785-794.

Stockman, A.L., Brancalion, P.H.S., Novembre, A.D.L.C. \& Chamma, H.M.C.P. 2007. Sementes de ipê-branco (Tabebuia roseo-alba (Ridl.) Sand. - Bignoniaceae): temperatura e substrato para o teste de germinação. Revista Brasileira de Sementes 29: 139-143.

Takaki, M. 2001. New proposal of classification of seeds based on forms of phytochrome instead of photoblastism. Brazilian Journal of Plant Physiology 13: 103-107.

Yu, Y., Baskin, J.M., Bakin, C.C., Tang, Y. \& Cao, M. 2008. Ecology of seed germination of eight non-pioneer tree species from a tropical seasonal rain forest in southwest china. Plant Ecology 197: 1-16. 